Research Article

\title{
Comparative Evaluation and Measure of Accuracy of ELISAs, CLIAs, and ECLIAs for the Detection of HIV Infection among Blood Donors in China
}

\author{
Le Chang, ${ }^{1,2}$ Junpeng Zhao $\mathbb{C D}^{1,2,3,4}$ Fei Guo, ${ }^{1,2}$ Huimin Ji, ${ }^{1,2,3}$ Lu Zhang, ${ }^{1,2,3}$ Xinyi Jiang, ${ }^{1,2,3}$ \\ and Lunan Wang ${ }^{1,2,3}$ \\ ${ }^{1}$ National Center for Clinical Laboratories, Beijing Hospital, National Center of Gerontology, Institute of Geriatric Medicine, \\ Chinese Academy of Medical Sciences, Beijing, China \\ ${ }^{2}$ Beijing Engineering Research Center of Laboratory Medicine, Beijing Hospital, Beijing, China \\ ${ }^{3}$ Graduate School, Peking Union Medical College, Chinese Academy of Medical Sciences, Beijing, China \\ ${ }^{4}$ Shenzhen Blood Center, Shenzhen, China
}

Correspondence should be addressed to Lunan Wang; lunan99@163.com

Received 6 October 2019; Revised 2 July 2020; Accepted 11 July 2020; Published 14 August 2020

Academic Editor: Lucia Lopalco

Copyright $\odot 2020$ Le Chang et al. This is an open access article distributed under the Creative Commons Attribution License, which permits unrestricted use, distribution, and reproduction in any medium, provided the original work is properly cited.

\begin{abstract}
Background. Enzyme-linked immunosorbent assay (ELISA) is the only serological method approved for blood screening in China. Automated chemiluminescence immunoassay (CLIA) and electrochemiluminescence immunoassay (ECLIA) had been used in clinical laboratories but not applied to screen HIV among blood donors. This study aimed to evaluate the performance of ELISA, CLIA, and ECLIA, focusing on the feasibility of CLIA/ECLIA for blood screening. Method. 1029 blood donations from 14 blood centers screened by ELISA were enrolled in the study. All plasma samples were tested by eight ELISA assays in 16 blood centers, followed by the detection of CLIA and ECLIA methods in the National Center for Clinical Laboratories (NCCL), further confirmed by nucleic acid testing (NAT) and Western blot (WB). Results. Of 1029 samples, 136 were confirmed as HIV positive. CLIA and ECLIA assay had similar sensitivities with ELISAs but showed higher specificity (CLIA: 99.1\%, 885/893; ECLIA: 99.0\%, 884/893), concordance rate (CLIA: 99.2\%, 1021/1029; ECLIA: 99.1\%, 1020/1029), and positive predictive value (PPV) (CLIA: 94.4\%, 136/144; ECLIA: 93.8\%, 136/145) than most of ELISA kits ( $>5$ ELISAs $)(P<0.05)$. Kappa values of CLIA (0.967) and ECLIA (0.963) were the highest among all the serologic assays. Among 451 samples with initial ELISA reactivity, 315 were negatives, of which 307 (97.5\%) and 306 (97.1\%) were detected as nonreactive by CLIA (8 nonspecific reactions) and ECLIA (9 nonspecific reactions), respectively. Conclusion. Compared with ELISA, CLIA and ECLIA are more specific and accurate in detecting HIV antibody/antigen and can keep more nonspecifically reactive donors detected by ELISA. CLIA and ECLIA can be used for the improvement of serological blood screening strategy to avoid the unnecessary loss of blood donors.
\end{abstract}

\section{Background}

Ever since the first identification of HIV infectious case in Ruili City, Yunnan province, in 1985, HIV has spread numerically and geographically throughout China. According to the updated report, approximately 849,602 people are living with HIV and 262,442 individuals died of HIV-associated diseases in China [1]. The epidemic of HIV has been shifted from high risk population into the general population, including blood donors in China [2,3]. The prevalence of HIV among blood donors in China is about 10.33 in 100,000 between 2000 and 2009 [4]. Therefore, HIV screening is essential to ensure the strict safety of blood supply.

At present, NAT and ELISA are routinely applied to screen HIV infection. ELISA is the only approved serological assay for HIV screening among blood donations in China. The assay detects the presence of a ligand (commonly a protein) in a liquid sample through a solid-phase enzyme immunoassay (EIA) using antibodies directed against the protein to be measured [5]. The amounts of HIV antigens or 
antibodies are measured by the intensity of color produced by reaction between conjugate and substrate. CLIA and ECLIA methods use recombinant HIV antigens or antibodies coated paramagnetic microparticles that bind to HIV antigens or antibodies in plasma labelled recombinant HIV antigens or antibodies coated particles as conjugate. Antibody or antigen concentration is determined by the emitted light of antigenantibody reaction and measured using light reader [6,7].

CLIA and ECLIA, including sample preprocessing system and result analysis system from the same manufacturers, are fully automated and self-contained platforms which minimize operator involvement and have good reproducibility and partly avoid the false positive/negative brought by operator factors [8-10]. However, even if using the same kit in automatic ELISA platform in different Chinese blood centers, the preprocessing system, incubation and washing system, and microplate reader could be completely different, due to the open system of ELISA devices. The assembled automatic ELISA system is hard to ensure the standardization of results between different blood centers. Hence, the suitability for ELISA is defined by different parameters and may be further affected by other factors which are based on open system. The diversity of the virus including the prevalence of groups and subtypes and circulating recombinant forms can influence the effectiveness of the diagnosis [11].

The current study aimed to compare the performance between eight ELISAs and CLIA/ECLIA and discuss whether CLIA/ECLIA can be used for blood screening in China.

\section{Materials and Methods}

2.1. HIV Confirmatory Algorithm and Study Design. From March 2015 to September 2015, 1029 blood donations detected as HIV reactive using one or two ELISAs which were collected from 14 blood centers or blood banks and were screened for HIV. The number of blood donations was equal to the number of blood donors. All blood donations underwent HIV confirmatory algorithm: all the initial negative samples were performed NAT in the National Center for Clinical Laboratories (NCCL), and initial positives were tested by Western blot (WB) by local centers for disease control and prevention (CDC); the samples with indeterminate results of WB were further confirmed using WB and NAT in NCCL. Then, all the samples were sent to 16 blood screening laboratories for ELISA evaluation using one or two of the eight assays with automation systems; furthermore, CLIA and ECLIA screening among all the blood donations were performed in NCCL (Table 1). The results of the same ELISA reagent used by different laboratories in the same sample were not shown. The final result of each ELISA assay among 1029 blood donations was determined by more than half the results of different blood centers using the same ELISA. The study route was shown in Figure 1.

2.2. Statistical Analyses. SPSS 21.0 software was used for statistical analysis. Chi-square tests were performed on all the examined outcomes to compare sensitivity, specificity, concordance rate, positive predictive value (PPV), and negative predictive value (NPV) between CLIA, ECLIA, and ELISAs; $P<0.05$ was considered statistically significant. Sensitivity was the proportion of true positives among donations with serologic reactivity; specificity was calculated using the number of true negatives divided by nonreactive results in serologic assays. Concordance rate (accuracy) will be performed and calculated through the percentage of true positives and negatives among 1029 blood donations. PPV and NPV are the proportions of positive and negative results in diagnostic tests that are true positive and true negative results, respectively.

\section{Results}

Of 1029 donations, 136 were confirmed as HIV positive and 893 were HIV negative. PPV of initial ELISA test $(30.2 \%)$ had a significant difference with CLIA (94.4\%) and ECLIA (93.8\%), respectively $(P<0.05)$ (Table 2). Among 451 samples with initial ELISA reactivity, 315 were negatives, of which 307 (97.5\%) and 306 (97.1\%) were detected as nonreactive by CLIA ( 8 nonspecific reactions) and ECLIA (9 nonspecific reactions), respectively.

As shown in Table 3, the sensitivity of CLIA or ECLIA had no significant difference with 8 ELISAs $(P<0.05)$, only two or three samples were missed by several ELISAs (not shown), but these samples were detected by CLIA, ECLIA, and NAT. The CLIA and ECLIA showed higher specificities and PPVs than most of ELISAs (Wantai, KHB, Bio-Rad, Wantai $\left[4^{\text {th }}\right]$, Murex $\left[4^{\text {th }}\right]$, Livzon $\left.\left[4^{\text {th }}\right]\right), P<0.05$. Collectively, no significant differences found in sensitivity and NPV between 8 ELISAs and CLIA/ECLIA $(P<0.05)$; Kappa values of CLIA (0.967) and ECLIA (0.963) were the highest among all the serologic assays. However, ELISA evaluations in some laboratories (HN, JN, LN) showed lower sensitivities and NPVs than CLIA and ECLIA $(P<0.05)$. InTec and Livzon ELISAs had similar specificities/PPVs/concordance rates to CLIA and ECLIA $(P<0.05)$. However, among the five HIV blood screening laboratories using InTec, ELISA results in HLJ and LN showed lower specificities and concordance rates, in comparison to CLIA/ECLIA $(P<0.05)$. In addition, CLIA and ECLIA had higher PPV and concordance rate than InTec ELISAs tested by LN and CQ, respectively, $P<0.05$. Besides, of 2 blood centers using Livzon, the ELISAs tested by $\mathrm{HZ}$ and $\mathrm{ZH}$ were less specific than CLIA/ECLIA, and ELISA screening in $\mathrm{ZH}$ had lower PPV than CLIA/ECLIA.

\section{Discussion}

ELISA is the only approved serological assay for HIV screening among blood donations in China. CLIA and ECLIA have been proved to be at least as specific and sensitive as common assays in serological detection of many infectious diseases [12-15] but not applied for HIV blood screening in China. CLIA and ECLIA have been used for routine screening among blood donors in the US [16], Australia [17], Sweden [18], and Italy [10] for many years. Hence, evaluation of ELISA, CLIA, and ECLIA among blood 
TABLE 1: Blood screening laboratories of study participation and their commercially available HIV serology kits.

\begin{tabular}{|c|c|c|c|c|c|}
\hline Code & $\begin{array}{c}\text { Blood screening } \\
\text { labs }\end{array}$ & $\begin{array}{l}\text { HIV reactive } \\
\quad(n=451)\end{array}$ & $\begin{array}{l}\text { HIV nonreactive } \\
(n=578)\end{array}$ & \multicolumn{2}{|c|}{ HIV serology kits } \\
\hline 1 & SZ & 73 & 50 & Bio-Rad HIV Ab-Ag $\left(4^{\text {th }}\right)$ & - \\
\hline 2 & $\mathrm{XY}$ & 14 & 9 & InTec HIV Ab & Wantai HIV Ab \\
\hline 3 & $\mathrm{HB}$ & 26 & 18 & Murex HIV Ab-Ag $\left(4^{\text {th }}\right)$ & Wantai HIV Ab-Ag $\left(4^{\text {th }}\right)$ \\
\hline 4 & JS & 32 & 50 & InTec HIV Ab & Wantai HIV Ab-Ag $\left(4^{\text {th }}\right)$ \\
\hline 5 & LN & 19 & 50 & KHB HIV Ab & InTec HIV Ab \\
\hline 6 & HLJ & 29 & 76 & Bio-Rad HIV Ab-Ag $\left(4^{\text {th }}\right)$ & InTec HIV Ab \\
\hline 7 & $\mathrm{SD}$ & 44 & 50 & InTec HIV Ab & Murex HIV Ab-Ag $\left(4^{\text {th }}\right)$ \\
\hline 8 & $\mathrm{TJ}$ & 70 & 49 & Murex HIV Ab-Ag $\left(4^{\text {th }}\right)$ & Wantai HIV Ab-Ag $\left(4^{\text {th }}\right)$ \\
\hline 9 & $\mathrm{HN}$ & 25 & 112 & Wantai HIV Ab & - \\
\hline 10 & CQ & 31 & 18 & Bio-Rad HIV Ab-Ag $\left(4^{\text {th }}\right)$ & InTec HIV Ab \\
\hline 11 & $\mathrm{YN}$ & 8 & 15 & Bio-Rad HIV Ab-Ag $\left(4^{\text {th }}\right)$ & KHB HIV Ab \\
\hline 12 & $\mathrm{JL}$ & 48 & 11 & KHB HIV Ab & Wantai HIV Ab-Ag $\left(4^{\text {th }}\right)$ \\
\hline 13 & $\mathrm{HZ}$ & 3 & 21 & Livzon HIV Ab & - \\
\hline 14 & $\mathrm{TZ}$ & 29 & 49 & Bio-Rad HIV Ab-Ag $\left(4^{\text {th }}\right)$ & Murex HIV Ab-Ag $\left(4^{\text {th }}\right)$ \\
\hline 15 & $\mathrm{BJ}$ & - & - & Bio-Rad HIV Ab-Ag ( $\left.4^{\text {th }}\right)$ & Wantai HIV Ab \\
\hline 16 & $\mathrm{ZH}$ & - & - & Livzon HIV Ab-Ag $\left(4^{\text {th }}\right)$ & - \\
\hline 17 & $\mathrm{JN}$ & - & - & KHB HIV Ab & Wantai HIV Ab-Ag $\left(4^{\text {th }}\right)$ \\
\hline 18 & $\mathrm{CZ}$ & - & - & Murex HIV Ab-Ag $\left(4^{\text {th }}\right)$ & - \\
\hline 19 & NCCL & - & - & $\begin{array}{l}\text { Abbott ARCHITECT HIV Ag/Ab } \\
\text { Combo }^{\S}\left(4^{\text {th }}\right)\end{array}$ & $\begin{array}{l}\text { Roche Elecsys HIV combi } \\
\operatorname{PT}^{\dagger}\left(4^{\text {th }}\right)\end{array}$ \\
\hline
\end{tabular}

a: ${ }^{\S}$ CLIA ${ }^{\dagger}$ ECLIA, and other HIV serology kits are ELISAs. b: $4^{\text {th }}$ : fourth-generation ELISA kits. Other HIV serology kits are third-generation ELISA kits. ELISAs: Wantai Diagnostic Kit for antibody to human immunodeficiency virus (Beijing Wantai Biological Pharmacy, Beijing, China) (Wantai), Wantai Diagnostic Kit for antibody and antigen to human immunodeficiency virus (Beijing Wantai Biological Pharmacy, Beijing, China) (Wantai (4 $\left.{ }^{\text {th }}\right)$ ), Beijing, China; KHB Diagnostic Kit for antibody to human immunodeficiency virus (Shanghai Kehua Bioengineering Co., Shanghai, China) (KHB), InTec Diagnostic Kit for antibody to human immunodeficiency virus (InTec Products, Inc., Xiamen, China) (InTec), Livzon Diagnostic Kit for antibody to human immunodeficiency virus (Zhuhai Livzon Diagnostics Inc., Zhuhai, China) (Livzon), Livzon Diagnostic Kit for antibody and antigen to human immunodeficiency virus (Zhuhai Livzon Diagnostics Inc., Zhuhai, China) (Livzon $\left(4^{\text {th }}\right)$ ), Murex HIV Ag/Ab combination (Diasorin, Saluggia, Italy) (Murex $\left(4^{\text {th }}\right)$ ), Bio-Rad GS HIV Combo Ag/Ab EIA (Bio-Rad, Marnes la Coquette, France) (Bio-Rad $\left(4^{\text {th }}\right)$ ). c: blood centers (codes 1-14) were sources of study samples. d: blood centers (codes 3-18) performed HIV screening for all the samples using one or two ELISA kits. CLIA and ECLIA were conducted by NCCL.

donations is critical for the development of HIV serological screening strategy. To date, this comparative study firstly investigated the performance of 8 ELISA kits (including all ELISA kits for HIV blood screening in China) for the detection of HIV and comparing CLIA, ECLIA, and 8 ELISAs among blood donors. The sources of study samples were comprehensive, including 14 blood screening laboratories. Additionally, less comparative evaluation measure of accuracy between CLIA, ECLIA, and ELISA among blood donors focused on the feasibility of CLIA/ECLIA for blood screening and the improvement of serological screening strategy in China.

Using one or two HIV ELISA kits followed with NAT were required by Chinese Blood Bank Technical Operation Procedure (2015 version) to screen HIV in blood banks. The majority of the samples that tested initial ELISA reactive for HIV were nonspecific reactivity in the present study. This phenomenon may be related to the low prevalence of HIV [19], apart from the factors of reagent and laboratories. The previous study reported that most of donations were discarded and donors were deferred for nonspecific reactivity [20]. According to an official investigation report about clinical blood transfusion, the growth rate of blood supply is still lower than the rate of growing clinical demand for transfusion [21]. ECLIA (97.1\%, 306/315) and CLIA $(97.5 \%, 307 / 315)$ methods could reduce $97 \%$ nonspecific reactions produced by
ELISAs (Table 2). Hence, CLIA and ECLIA can be used for blood screening to avoid unnecessary loss of blood donations and also can be used for blood reentry [22].

In the study, we observed a high agreement in HIV antigen/antibody screening between CLIA and ECLIA, similar to Italy's study [10]. Previous studies indicated CLIA with high sensitivity, specificity, and high degree of automation for HIV antibody-antigen testing can be used for the detection of HIV infection $[15,23,24] .4^{\text {th }}$ generation serological assays can detect HIV antigen and antibody, while $3^{\text {rd }}$ generation serological assays only detect HIV antibody. The results showed that the specificity, concordance rate, and PPV of $3^{\text {rd }}$ generation ELISA kits were higher than $4^{\text {th }}$ generation ELISA kits, possibly due to higher sensitivity and nonspecific reactivity of $4^{\text {th }}$ generation ELISA kits $[25,26]$. However, CLIA and ECLIA, as $4^{\text {th }}$ generation serological assays, are more specific and accurate to detect HIV antigen/ antibody among blood donations, compared with the most of ELISAs ( ${ }^{\text {rd }}$ and $4^{\text {th }}$ generation ELISA). Note that specificity of CLIA and ECLIA was significantly higher than most of the ELISA kits, which demonstrated that ELISA may lead to more loss of donations due to the nonspecific reactions.

Among eight ELISAs, only InTec and Livzon were not different from CLIA/ECLIA about specificity/PPV/concordance rate. Nevertheless, InTec ELISAs tested by HLJ, LN, and CQ and Livzon ELISAs tested by HZ and ZH had lower specificities, PPVs, and/or concordance rates than ECLIA 


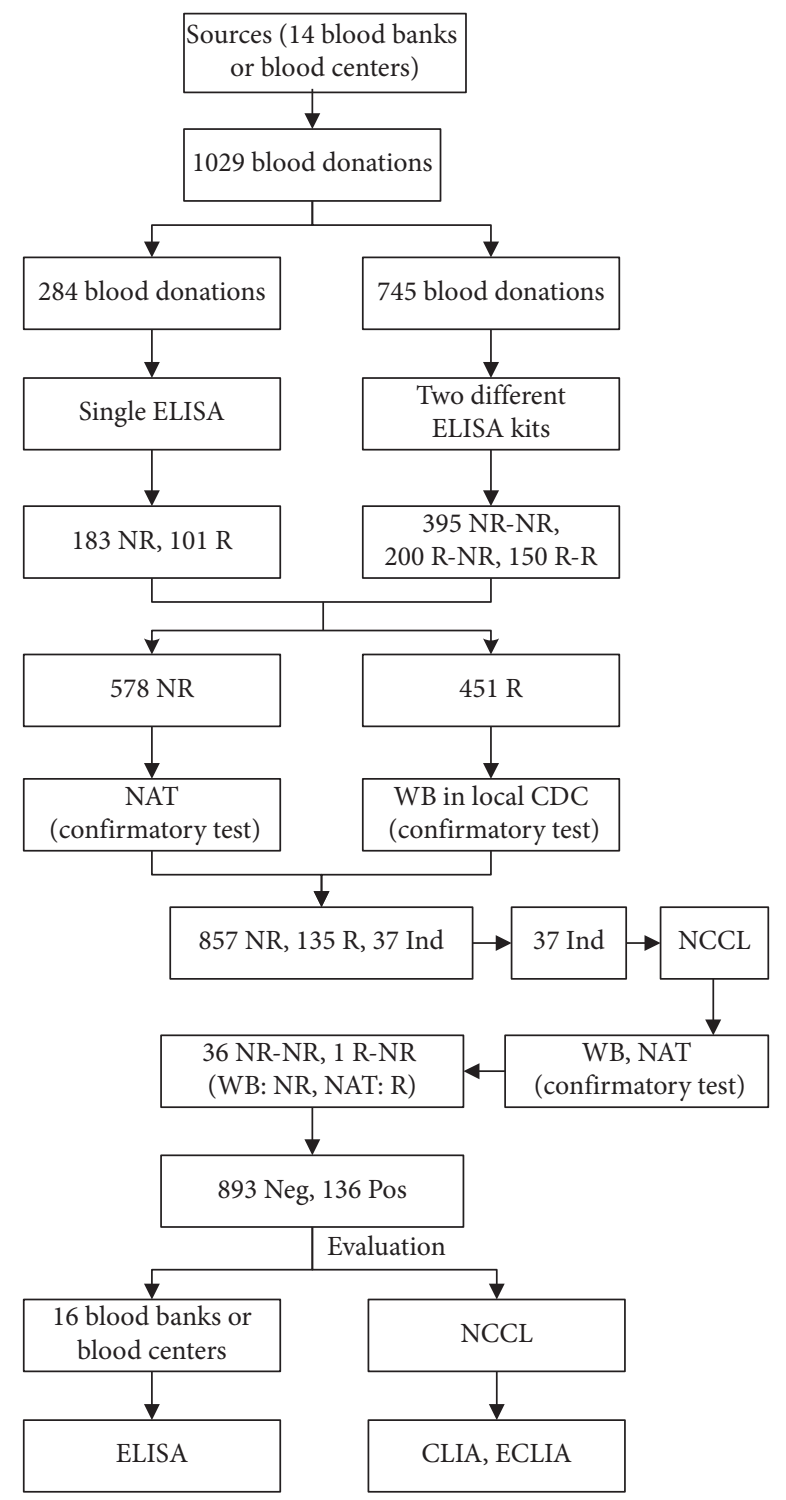

FIgURE 1: The study route. NR: nonreactive, R: reactive, Pos: positive, Neg: negative, WB: Western blot, CLIA: Abbott ARCHITECT HIV Ag/Ab Combo, ECLIA: Roche Elecsys HIV combi PT, and NAT: nucleic acid testing of Cobas TaqScreen MPX test v2.0.

and CLIA, suggesting that using the same ELISA reagent to test the same sample in different laboratories may produce different results. It indicated that the open system assay (ELISA) may lead to less than optimum results and the performance of the assay being evaluated is considered to be lower than it actually is, due to the requirement of high level of operator involvement and the overall performance of the assay itself $[27,28]$. The assembly automatic ELISA system may lead to bias of result in the same ELISA kit and the same sample between different laboratories. ELISA kits used in the blood centers can present a great variability depending on the supplier. For CLIA and ECLIA methods, there are few opportunities for error; the systems and assays can only be run as intended by the manufacturer, controlled by the system software.

In summary, significant differences in sensitivity, specificity, concordance rate, PPV, and NPV between some
ELISAs and CLIA/ECLIA were found in several blood centers.

At present, all blood centers must use ELISA as serological assay for HIV blood screening; the performance of ELISA about sensitivity was decent for blood supply. Only two or three positive samples were missed by ELISAs, while none was missed by NAT. Consequently, HIV screening strategy in China can ensure the blood safety. The sensitivity of ELISA met the detection of HIV among blood donors, which is the primary factor of blood screening assay, but specificity and feasibility must also be considered, the balance point between sensitivity, specificity, and feasibility being determined by each transfusion service. CLIA and ECLIA had been used in clinical laboratories in China, with higher specificity and lower operator involvement than ELISA, but the higher cost of CLIA and ECLIA limited their application in blood centers. However, 


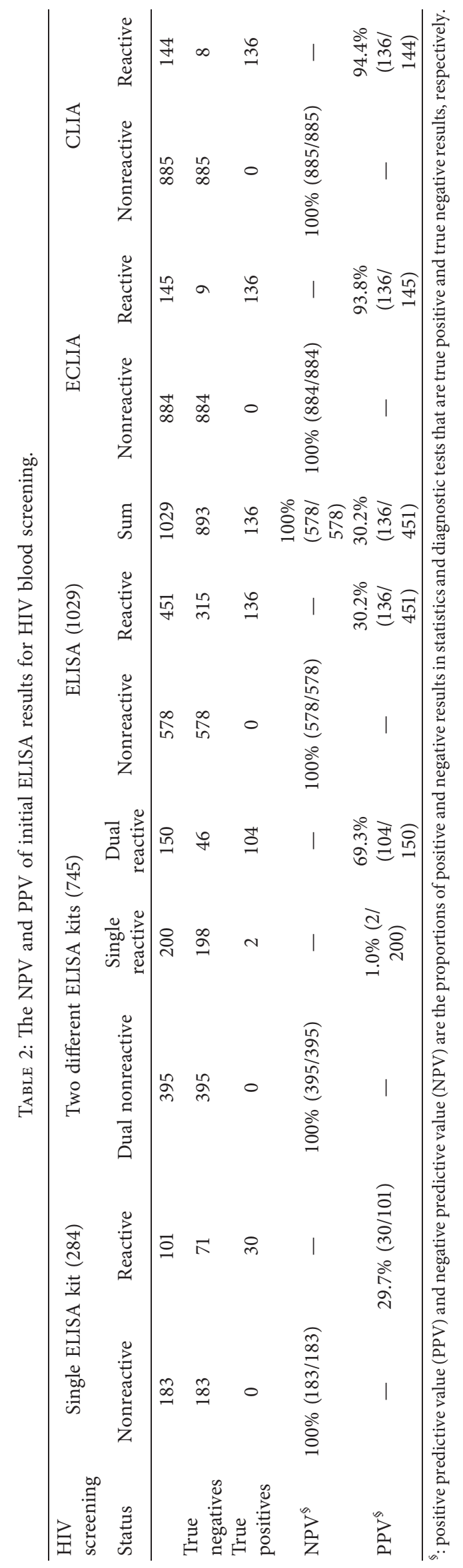


TABLE 3: The performance of serology kits for HIV screening ${ }^{\S}$.

\begin{tabular}{|c|c|c|c|c|c|c|c|}
\hline $\begin{array}{l}\text { Blood } \\
\text { screening } \\
\text { laboratories }\end{array}$ & $\begin{array}{l}\text { HIV } \\
\text { serology } \\
\text { kits }\end{array}$ & $\begin{array}{c}\text { Sensitivity } \\
n=136 \text { (\%), } \\
95 \% \text { CI }\end{array}$ & $\begin{array}{c}\text { Specificity } n=893 \\
(\%), 95 \% \mathrm{CI}\end{array}$ & $\begin{array}{c}\text { Concordance rate }{ }^{\| l} \\
n=1029(\%), 95 \% \\
\text { CI }\end{array}$ & PPV, 95\% CI & NPV, 95\% CI & $\underset{(\kappa)}{\text { Kappa }}$ \\
\hline BJ & Wantai & $\begin{array}{c}134(98.5 \%), \\
96.5 \%-100.5 \%\end{array}$ & $\begin{array}{c}859(96.2 \%)^{* * \dagger \dagger} \\
95.0 \%-97.4 \%\end{array}$ & $\begin{array}{c}993(96.5 \%)^{* * \dagger \dagger} \\
95.4 \%-97.6 \%\end{array}$ & $\begin{array}{c}79.8 \%^{* * \dagger \dagger}, \\
73.7 \%-85.8 \%\end{array}$ & $99.8 \%, 99.4 \%-100.1 \%$ & 0.861 \\
\hline \multirow[t]{2}{*}{ HN } & Wantai & $\begin{array}{l}131(96.3 \%)^{* \dagger} \\
93.1 \%-99.5 \%\end{array}$ & $\begin{array}{c}839(94.0 \%)^{* * \dagger \dagger} \\
92.5 \%-95.5 \%\end{array}$ & $\begin{array}{c}970(94.3 \%)^{* * \dagger \dagger} \\
92.9 \%-95.7 \%\end{array}$ & $\begin{array}{c}70.8 \%^{* * \dagger \dagger} \\
64.3 \%-77.3 \%\end{array}$ & $99.4 \%{ }^{* \dagger}, 98.9 \%-99.9 \%$ & 0.783 \\
\hline & Wantai $^{\S}$ & $\begin{array}{c}134(98.5 \%) \\
96.5 \%-100.5 \%\end{array}$ & $\begin{array}{c}861(96.4 \%)^{* * \dagger \dagger}, \\
95.2 \%-97.6 \%\end{array}$ & $\begin{array}{c}995(96.7 \%)^{* * \dagger \dagger} \\
95.6 \%-97.8 \%\end{array}$ & $\begin{array}{c}80.7 \% \%^{* * \dagger \dagger} \\
74.8 \%-86.7 \%\end{array}$ & $99.8 \%, 99.4 \%-100.1 \%$ & 0.868 \\
\hline YN & KHB & $\begin{array}{c}134(98.5 \%) \\
96.5 \%-100.5 \%\end{array}$ & $\begin{array}{l}872(97.6 \%)^{* \dagger}, \\
96.6 \%-98.6 \%\end{array}$ & $\begin{array}{c}1006(97.8 \%)^{* \dagger \dagger} \\
96.9 \%-98.7 \%\end{array}$ & $\begin{array}{c}86.5 \%^{* \dagger} \\
81.1 \%-91.8 \%\end{array}$ & $99.8 \%, 99.5 \%-100.1 \%$ & 0.908 \\
\hline JN & KHB & $\begin{array}{l}131(96.3 \%)^{* \dagger}, \\
93.1 \%-99.5 \%\end{array}$ & $\begin{array}{c}868(97.2 \%)^{* * \dagger \dagger} \\
96.1 \%-98.3 \%\end{array}$ & $\begin{array}{c}999(97.1 \%)^{* * \dagger \dagger} \\
96.1 \%-98.1 \%\end{array}$ & $\begin{array}{c}84.0 \%^{* * \dagger \dagger} \\
78.2 \%-89.7 \%\end{array}$ & $99.4 \%^{* \dagger}, 98.9 \%-99.9 \%$ & 0.880 \\
\hline \multirow[t]{2}{*}{ LN } & KHB & $\begin{array}{c}129(94.9 \%)^{* * \dagger \dagger} \\
91.2 \%-98.6 \%\end{array}$ & $\begin{array}{c}843(94.4 \%)^{* * \dagger \dagger} \\
92.9 \%-95.9 \%\end{array}$ & $\begin{array}{c}972(94.5 \%)^{* * \dagger \dagger} \\
95.4 \%-97.6 \%\end{array}$ & $\begin{array}{c}72.1 \% \%^{* * \dagger \dagger} \\
65.5 \%-78.6 \%\end{array}$ & $\begin{array}{c}99.2 \%^{* *+\dagger}, 98.6 \%- \\
99.8 \%\end{array}$ & 0.787 \\
\hline & $\mathrm{KHB}^{\S}$ & $\begin{array}{c}134(98.5 \%) \\
96.5 \%-100.5 \%\end{array}$ & $\begin{array}{c}873(97.8 \%)^{* \dagger} \\
96.8 \%-98.8 \%\end{array}$ & $\begin{array}{c}1007(97.9 \%)^{* \dagger} \\
97.0 \%-98.8 \%\end{array}$ & $\begin{array}{c}87.0 \%{ }^{* \dagger} \\
81.7 \%-92.3 \%\end{array}$ & $99.8 \%, 99.5 \%-100.1 \%$ & 0.912 \\
\hline SD & InTec & $\begin{array}{c}133(97.8 \%) \\
95.3 \%-100.3 \%\end{array}$ & $\begin{array}{l}879(98.4 \%) \\
97.6 \%-99.2 \%\end{array}$ & $\begin{array}{l}1012(98.3 \%) \\
97.5 \%-99.1 \%\end{array}$ & $\begin{array}{c}90.5 \%, \\
85.8 \%-95.2 \%\end{array}$ & $99.7 \%, 99.3 \%-100.0 \%$ & 0.930 \\
\hline JS & InTec & $\begin{array}{c}133(97.8 \%) \\
95.3 \%-100.3 \%\end{array}$ & $\begin{array}{l}880(98.5 \%) \\
97.7 \%-99.3 \%\end{array}$ & $\begin{array}{l}1013(98.4 \%), \\
97.6 \%-99.2 \%\end{array}$ & $\begin{array}{c}91.1 \% \\
86.5 \%-95.7 \%\end{array}$ & $99.7 \%, 99.3 \%-100.0 \%$ & 0.934 \\
\hline HLJ & InTec & $\begin{array}{c}133(97.8 \%), \\
95.3 \%-100.3 \%\end{array}$ & $\begin{array}{l}875(98.0 \%)^{\dagger}, \\
97.1 \%-98.9 \%\end{array}$ & $\begin{array}{c}1008(98.0 \%)^{* \dagger} \\
97.1 \%-98.9 \%\end{array}$ & $\begin{array}{c}88.1 \% \\
82.9 \%-93.2 \%\end{array}$ & $99.7 \%, 99.3 \%-100.0 \%$ & 0.915 \\
\hline $\mathrm{LN}$ & InTec & $\begin{array}{l}132(97.1 \%)^{* \dagger}, \\
94.3 \%-99.9 \%\end{array}$ & $\begin{array}{c}872(97.6 \%)^{* \dagger}, \\
96.6 \%-98.6 \%\end{array}$ & $\begin{array}{c}1004(97.6 \%)^{* * * \dagger} \\
96.7 \%-98.5 \%\end{array}$ & $\begin{array}{c}86.3 \%^{* \dagger} \\
80.8 \%-91.7 \%\end{array}$ & $99.5 \%, 99.1 \%-100.0 \%$ & 0.899 \\
\hline \multirow[t]{2}{*}{ CQ } & InTec & $\begin{array}{c}133(97.8 \%), \\
95.3 \%-100.3 \%\end{array}$ & $\begin{array}{l}876(98.1 \%), \\
97.2 \%-99.0 \%\end{array}$ & $\begin{array}{c}1009(98.1 \%)^{* \dagger} \\
97.3 \%-98.9 \%\end{array}$ & $\begin{array}{c}88.7 \% \\
83.6 \%-93.7 \%\end{array}$ & $99.7 \%, 99.3 \%-100.0 \%$ & 0.919 \\
\hline & $\operatorname{InTec}{ }^{\mathfrak{S}}$ & $\begin{array}{c}133(97.8 \%), \\
95.3 \%-100.3 \%\end{array}$ & $\begin{array}{l}881(98.7 \%) \\
98.0 \%-99.4 \%\end{array}$ & $\begin{array}{l}1014(98.5 \%) \\
97.8 \%-99.2 \%\end{array}$ & $\begin{array}{c}91.7 \%, \\
87.3 \%-96.2 \%\end{array}$ & 99.7\%, 99.3\%-100.0\% & 0.938 \\
\hline $\mathrm{HZ}$ & Livzon & $\begin{array}{c}133(97.8 \%), \\
95.3 \%-100.3 \%\end{array}$ & $\begin{array}{l}875(98.0 \%)^{\dagger} \\
97.1 \%-98.9 \%\end{array}$ & $\begin{array}{c}1008(98.0 \%)^{* \dagger} \\
97.1 \%-98.9 \%\end{array}$ & $\begin{array}{c}88.1 \% \\
82.9 \%-93.2 \%\end{array}$ & $99.7 \%, 99.3 \%-100.0 \%$ & 0.915 \\
\hline \multirow[t]{2}{*}{$\mathrm{ZH}$} & Livzon & $\begin{array}{c}133(97.8 \%) \\
95.3 \%-100.3 \%\end{array}$ & $\begin{array}{c}873(97.8 \%)^{* \dagger}, \\
96.8 \%-98.8 \%\end{array}$ & $\begin{array}{c}1006(97.8 \%)^{* \dagger \dagger} \\
96.9 \%-98.7 \%\end{array}$ & $\begin{array}{c}86.9 \%^{* \dagger} \\
81.6 \%-92.2 \%\end{array}$ & $99.7 \%, 99.3 \%-100.0 \%$ & 0.907 \\
\hline & Livzon $^{\S}$ & $\begin{array}{c}133(97.8 \%) \\
95.3 \%-100.3 \%\end{array}$ & $\begin{array}{l}876(98.1 \%) \\
97.2 \%-99.0 \%\end{array}$ & $\begin{array}{c}1009(98.1 \%)^{* \dagger} \\
97.3 \%-98.9 \%\end{array}$ & $\begin{array}{c}88.7 \% \\
83.6 \%-93.7 \%\end{array}$ & $99.7 \%, 99.3 \%-100.0 \%$ & 0.919 \\
\hline SD & $\begin{array}{l}\text { Bio-Rad } \\
\left(4^{\text {th }}\right)\end{array}$ & $\begin{array}{c}136(100 \%) \\
100.0 \%-100.0 \%\end{array}$ & $\begin{array}{c}795(89.0 \%)^{* * \dagger \dagger} \\
87.0 \%-91.0 \%\end{array}$ & $\begin{array}{c}931(90.5 \%)^{* * \dagger \dagger} \\
88.7 \%-92.3 \%\end{array}$ & $\begin{array}{l}58.1 \%{ }^{* * \dagger \dagger} \\
51.8 \%-64.4 \%\end{array}$ & $100 \%, 100.0 \%-100.0 \%$ & 0.682 \\
\hline SZ & $\begin{array}{l}\text { Bio-Rad } \\
\left(4^{\text {th }}\right)\end{array}$ & $\begin{array}{c}136(100 \%) \\
100.0 \%-100.0 \%\end{array}$ & $\begin{array}{c}795(89.0 \%)^{* * \dagger \dagger} \\
87.0 \%-91.0 \%\end{array}$ & $\begin{array}{c}931(90.5 \%)^{* * \dagger \dagger}, \\
88.7 \%-92.3 \%\end{array}$ & $\begin{array}{c}58.1 \%^{* * \dagger \dagger} \\
51.8 \%-64.4 \%\end{array}$ & $100 \%, 100.0 \%-100.0 \%$ & 0.682 \\
\hline BJ & $\begin{array}{l}\text { Bio-Rad } \\
\left(4^{\text {th }}\right)\end{array}$ & $\begin{array}{c}136(100 \%) \\
100.0 \%-100.0 \%\end{array}$ & $\begin{array}{c}798(89.4 \%)^{* * \dagger \dagger} \\
87.4 \%-91.4 \%\end{array}$ & $\begin{array}{c}934(90.8 \%)^{* * \dagger \dagger} \\
89.0 \%-92.6 \%\end{array}$ & $\begin{array}{c}58.9 \%^{* * \dagger \dagger} \\
52.6 \%-65.2 \%\end{array}$ & $100 \%, 100.0 \%-100.0 \%$ & 0.689 \\
\hline \multirow[t]{2}{*}{ HLJ } & $\begin{array}{l}\text { Bio-Rad } \\
\quad\left(4^{\text {th }}\right)\end{array}$ & $\begin{array}{c}136(100 \%) \\
100.0 \%-100.0 \%\end{array}$ & $\begin{array}{c}797(89.2 \%)^{* * \dagger \dagger} \\
87.2 \%-91.2 \%\end{array}$ & $\begin{array}{c}933(90.7 \%)^{* * \dagger \dagger} \\
88.9 \%-92.5 \%\end{array}$ & $\begin{array}{c}58.6 \%^{* * \dagger \dagger} \\
52.3 \%-64.9 \%\end{array}$ & $100 \%, 100.0 \%-100.0 \%$ & 0.687 \\
\hline & $\begin{array}{l}\text { Bio-Rad } \\
\left(4^{\text {th }}\right)^{\S}\end{array}$ & $\begin{array}{c}136(100 \%) \\
100.0 \%-100.0 \%\end{array}$ & $\begin{array}{c}812(90.9 \%)^{* * \dagger \dagger} \\
89.0 \%-92.8 \%\end{array}$ & $\begin{array}{c}948(92.1 \%)^{* * \dagger \dagger} \\
90.5 \%-93.7 \%\end{array}$ & $\begin{array}{c}62.7 \%^{* * \dagger \dagger} \\
56.3 \%-69.1 \%\end{array}$ & $100 \%, 100.0 \%-100.0 \%$ & 0.726 \\
\hline $\mathrm{JL}$ & $\begin{array}{c}\text { Wantai } \\
\left(4^{\text {th }}\right)\end{array}$ & $\begin{array}{c}135(99.3 \%) \\
97.9 .0 \%-100.7 \%\end{array}$ & $\begin{array}{c}843(94.4 \%)^{* * \dagger \dagger} \\
92.9 \%-95.9 \%\end{array}$ & $\begin{array}{c}978(95.0 \%)^{* * \dagger \dagger} \\
93.7 \%-96.3 \%\end{array}$ & $\begin{array}{c}73.0 \% \%^{* * \dagger \dagger} \\
66.6 \%-79.3 \%\end{array}$ & $99.9 \%, 99.7 \%-100.1 \%$ & 0.813 \\
\hline JS & $\begin{array}{l}\text { Wantai } \\
\left(4^{\text {th }}\right)\end{array}$ & $\begin{array}{c}135(99.3 \%) \\
97.9 .0 \%-100.7 \%\end{array}$ & $\begin{array}{c}863(96.6 \%)^{* * \dagger \dagger} \\
95.4 \%-97.8 \%\end{array}$ & $\begin{array}{c}998(97.0 \%)^{* * \dagger \dagger} \\
96.0 \%-98.0 \%\end{array}$ & $\begin{array}{c}81.8 \% \%^{* * \dagger} \\
76.0 \%-87.7 \%\end{array}$ & $99.9 \%, 99.7 \%-100.1 \%$ & 0.88 \\
\hline $\mathrm{TJ}$ & $\begin{array}{l}\text { Wantai } \\
\left(4^{\text {th }}\right)\end{array}$ & $\begin{array}{c}133(97.8 \%) \\
95.3 \%-100.3 \%\end{array}$ & $\begin{array}{c}853(95.5 \%)^{* * \dagger \dagger} \\
94.1 \%-96.9 \%\end{array}$ & $\begin{array}{c}986(95.8 \%)^{* * \dagger \dagger} \\
94.6 \%-97.0 \%\end{array}$ & $\begin{array}{c}76.9 \%^{* * \dagger \dagger} \\
70.6 \%-83.1 \%\end{array}$ & $99.6 \%, 99.3 \%-100.0 \%$ & 0.837 \\
\hline \multirow[t]{2}{*}{$\mathrm{HB}$} & $\begin{array}{l}\text { Wantai } \\
\left(4^{\text {th }}\right)\end{array}$ & $\begin{array}{c}134(98.5 \%), \\
96.5 \%-100.5 \%\end{array}$ & $\begin{array}{c}854(95.6 \%)^{* * \dagger \dagger} \\
94.3 \%-96.9 \%\end{array}$ & $\begin{array}{c}988(96.0 \%)^{* * \dagger \dagger} \\
94.8 \%-97.2 \%\end{array}$ & $\begin{array}{c}77.5 \% \%^{* * \dagger} \\
71.3 \%-83.7 \%\end{array}$ & $99.8 \%, 99.4 \%-100.1 \%$ & 0.844 \\
\hline & $\begin{array}{l}\text { Wantai } \\
\left(4^{\text {th }}\right)^{\S}\end{array}$ & $\begin{array}{c}135(99.3 \%) \\
97.9 .0 \%-100.7 \%\end{array}$ & $\begin{array}{c}863(96.6 \%)^{* *+\dagger} \\
95.4 \%-97.8 \%\end{array}$ & $\begin{array}{c}998(97.0 \%)^{* * \dagger \dagger} \\
96.0 \%-98.0 \%\end{array}$ & $\begin{array}{c}81.8 \% \%^{* * \dagger} \\
76.0 \%-87.7 \%\end{array}$ & $99.9 \%, 99.7 \%-100.1 \%$ & 0.880 \\
\hline $\mathrm{TZ}$ & $\begin{array}{l}\text { Murex } \\
\left(4^{\text {th }}\right)\end{array}$ & $\begin{array}{c}135(99.3 \%) \\
97.9 .0 \%-100.7 \%\end{array}$ & $\begin{array}{c}833(93.3 \%)^{* *{ }^{\dagger \dagger}} \\
91.7 \%-94.9 \%\end{array}$ & $\begin{array}{c}968(94.1 \%)^{* * \dagger \dagger} \\
92.7 \%-95.5 \%\end{array}$ & $\begin{array}{c}69.2 \%^{* *+\dagger} \\
62.8 \%-75.7 \%\end{array}$ & $99.9 \%, 99.6 \%-100.1 \%$ & 0.782 \\
\hline $\mathrm{CZ}$ & $\begin{array}{l}\text { Murex } \\
\left(4^{\text {th }}\right)\end{array}$ & $\begin{array}{c}135(99.3 \%) \\
97.9 .0 \%-100.7 \%\end{array}$ & $\begin{array}{c}834(93.4 \%)^{* * \dagger \dagger} \\
91.8 \%-95.0 \%\end{array}$ & $\begin{array}{c}969(94.2 \%)^{* * \dagger \dagger} \\
92.8 \%-95.6 \%\end{array}$ & $\begin{array}{c}69.6 \%^{* *+\dagger} \\
63.1 \%-76.0 \%\end{array}$ & $99.9 \%, 99.6 \%-100.1 \%$ & 0.785 \\
\hline $\mathrm{HB}$ & $\begin{array}{l}\text { Murex } \\
\left(4^{\text {th }}\right)\end{array}$ & $\begin{array}{c}135(99.3 \%) \\
97.9 .0 \%-100.7 \%\end{array}$ & $\begin{array}{c}849(95.1 \%)^{* *+\dagger} \\
93.7 \%-96.5 \%\end{array}$ & $\begin{array}{c}984(95.6 \%)^{* * \dagger \dagger} \\
94.4 \%-96.8 \%\end{array}$ & $\begin{array}{c}75.4 \%^{* *+\dagger} \\
69.1 \%-81.7 \%\end{array}$ & 99.9\%, 99.7\%-100.1\% & 0.832 \\
\hline
\end{tabular}


TABLE 3: Continued.

\begin{tabular}{|c|c|c|c|c|c|c|c|}
\hline $\begin{array}{l}\text { Blood } \\
\text { screening } \\
\text { laboratories } \\
\end{array}$ & $\begin{array}{l}\text { HIV } \\
\text { serology } \\
\text { kits } \\
\end{array}$ & $\begin{array}{c}\text { Sensitivity } \\
n=136(\%), \\
95 \% \text { CI }\end{array}$ & $\begin{array}{c}\text { Specificity } n=893 \\
(\%), 95 \% \text { CI }\end{array}$ & $\begin{array}{c}\text { Concordance rate } \\
n=1029(\%), 95 \% \\
\text { CI }\end{array}$ & PPV, 95\% CI & NPV, 95\% CI & $\underset{(\kappa)}{\text { Kappa }}$ \\
\hline & $\underset{\left(4^{\text {th }}\right)^{\S}}{\text { Murex }}$ & $\begin{array}{c}135(99.3 \%) \\
97.9 .0 \%-100.7 \%\end{array}$ & $\begin{array}{c}842(94.3 \%)^{* * \dagger \dagger} \\
92.8 \%-95.8 \%\end{array}$ & $\begin{array}{c}977(94.9 \%)^{* *{ }^{\dagger \dagger}}, \\
93.6 \%-96.2 \%\end{array}$ & $\begin{array}{c}72.6 \% \%^{* * \dagger \dagger} \\
66.2 \%-79.0 \%\end{array}$ & 99.9\%, 99.7\%-100.1\% & 0.809 \\
\hline $\mathrm{ZH}$ & $\begin{array}{l}\text { Livzon } \\
\left(4^{\text {th }}\right)\end{array}$ & $\begin{array}{c}135(99.3 \%) \\
97.9 .0 \%-100.7 \%\end{array}$ & $\begin{array}{c}869(97.3 \%)^{* * \dagger \dagger} \\
96.2 \%-98.4 \%\end{array}$ & $\begin{array}{c}1004(97.6 \%)^{* * * \dagger} \\
96.7 \%-98.5 \%\end{array}$ & $\begin{array}{c}84.90 \% *^{* \dagger \dagger} \\
79.4 \%-90.4 \%\end{array}$ & $99.90 \%, 99.7 \%-100.1 \%$ & 0.901 \\
\hline NCCL & ECLIA & $\begin{array}{c}136(100 \%) \\
100.0 \%-100.0 \%\end{array}$ & $\begin{array}{l}884(99.0 \%) \\
98.4 \%-99.6 \%\end{array}$ & $\begin{array}{l}1020(99.1 \%) \\
98.5 \%-99.7 \%\end{array}$ & $\begin{array}{c}93.8 \%, \\
89.9 \%-97.7 \%\end{array}$ & $100 \%, 100.0 \%-100.0 \%$ & 0.963 \\
\hline NCCL & CLIA & $\begin{array}{c}136(100 \%) \\
100.0 \%-100.0 \%\end{array}$ & $\begin{array}{l}885(99.1 \%) \\
98.5 \%-99.7 \%\end{array}$ & $\begin{array}{l}1021(99.2 \%) \\
98.7 \%-99.7 \%\end{array}$ & $\begin{array}{c}94.4 \%, \\
90.7 \%-98.2 \%\end{array}$ & $100 \%, 100.0 \%-100.0 \%$ & 0.967 \\
\hline
\end{tabular}

${ }^{\$}$ The final result of each ELISA among 1029 blood donations was determined by more than half results of different blood centers using the same ELISA and S/ $\mathrm{CO}$ if the number of reactive and nonreactive results was equal. "The proportion of true results among samples screened by HIV serology kits. ${ }^{*} P<0.05$, ${ }^{* *} P<0.01,{ }^{\dagger} P<0.05,{ }^{\dagger \dagger} P<0.01$, ELISA, and ECLIA: the values $\left({ }^{*}\right)$ were compared with ECLIA (Roche Elecsys HIV combi PT); ELISA and CLIA: the values $\left({ }^{\dagger}\right)$ were compared with CLIA (Abbott ARCHITECT HIV Ag/Ab Combo).

the benefit of them for the health system must be taken into account, since their sensitivity and efficiency have been scientifically proven. After large-scale industrial production and policy support, the cost of CLIA/ECLIA will be under control. Furthermore, the parts of automatic ELISA system were different from ELISA manufacturers and blood centers, which were hard to standardize. In the future, CLIA or ECLIA may be approved by local authorities and used as a second confirmation step or routine screening for the detection of HIV infections among Chinese blood donors. If not all laboratories can use these techniques, centralized labs could perform the tests.

\section{Conclusions}

In summary, compared with ELISA, CLIA and ECLIA of full automation and closed system are more specific and accurate to detect HIV antibody/antigen among blood donations, which can keep a large number of blood donations with nonspecific ELISA reactions. The feasibility of CLIA and ECLIA is decent for blood screening, which may be approved as serological screening tool among blood donations to avoid the unnecessary loss of blood donors in China.

The limitation of the research is that CLIA/ECLIA and ELISA cannot simultaneously be performed by blood centers, due to no CLIA and ECLIA platform in blood centers. Comprehensive and comparative study of HIV serological screening about ELISA, CLIA, and ECLIA (CLIA and ECLIA: Including Abbott, Roche, and domestic manufacturers) among blood donors in China is already in progress.

\section{Abbreviations}

HIV: Human immunodeficiency virus

ELISA: Enzyme-linked immunosorbent assay

CLIA: Chemiluminescence immunoassay

ECLIA: Electrochemiluminescence immunoassay

NCCL: National Center for Clinical Laboratories

NAT: $\quad$ Nucleic acid testing

WB: Western blot
AIDS/ Acquired immunodeficiency syndrome/sexually

STD: $\quad$ transmitted disease

EIA: $\quad$ Enzyme immunoassay

HBV: Hepatitis B virus

HCV: Hepatitis $C$ virus

ALT: $\quad$ Alanine transaminase

S/CO: $\quad$ Signal to cut-off values

PPV: $\quad$ Positive predictive value

NPV: $\quad$ Negative predictive value

CDC: $\quad$ Center for Disease Control and Prevention

NAT: Nucleic acid testing.

\section{Data Availability}

The data used to support this study are available from the corresponding author upon request.

\section{Ethical Approval}

The institutional review board of the Beijing Hospital has approved the study. The methods in the study were in accordance with the guidelines of the Declaration of Helsinki.

\section{Consent}

Written informed consent was obtained from all subjects participating in this research.

\section{Conflicts of Interest}

The authors declare that they have no conflicts of interest.

\section{Authors' Contributions}

JZ and LC were joint first authors and contributed equally to this study. LW, JZ, and LC designed the study. JZ, LC, and FG conducted the laboratory tests. JZ, HJ, LZ, and XJ collected and analyzed the data and prepared the manuscript. LW and LC edited and reviewed the manuscript. All the authors critically reviewed and revised the manuscript drafts, approved the final version of the manuscript, and take responsibility for the integrity of the data and accuracy of 
data analysis. Le Chang and Junpeng Zhao contributed equally to this work.

\section{Acknowledgments}

The authors thank the clinical laboratory directors, Hongwei Ge, Wei Han, Chuanxing Zhang, Jinfeng Zeng, Wei Li, Yanhua Shi, Shaowen Zhu, Weiqing Zhu, Tong Pan, Haifeng Zhu, Fang Wang, Jianhua Fang, and Wei Zheng, from BJ, HB, SD, SZ, CQ, XY, JS, JN, SD, LN, HN, and HLJ blood centers, respectively, for kindly participating in sample collection and testing. This work was supported by grants from National Science and Technology Major Project of the Ministry of Science and Technology of China (2018ZX10306412-004 and 2018ZX10732101-003-002).

\section{References}

[1] Chinese Center for Disease Control and Prevention, "National center for AIDS/STD control and prevention, national AIDS/STD epidemic in December of 2017," Chinese Journal of AIDS \& STD, vol. 24, no. 2, p. 111, 2018.

[2] J. Wang, J. Liu, Y. Huang et al., "An analysis of risk factors for human immunodeficiency virus infection among Chinese blood donors," Transfusion, vol. 53, no. 10pt2, pp. 2431-2440, 2013.

[3] J. Wang, J. Liu, F. Yao et al., "Prevalence, incidence, and residual risks for transfusion-transmitted human immunodeficiency virus Types 1 and 2 infection among Chinese blood donors," Transfusion, vol. 53, no. 6, pp. 1240-1249, 2013.

[4] H. Xia, W. Qiang, T. Weiguo, and L. Hongwen, "A systematic review of HIV infection prevalence among volunteer blood donors in China," Chinese Journal of Blood Transfusion, vol. 25, no. s1, pp. 152-153, 2012.

[5] G. Weiland, "The enzyme-linked immunosorbent assay (ELISA)-a new serodiagnostic method for the detection of parasitic infections (author's transl)," MMW Munch Med Wochenschr, vol. 120, no. 44, pp. 1457-1460, 1978.

[6] W. R. Seitz, "Immunoassay labels based on chemiluminescence and bioluminescence," Clinical Biochemistry, vol. 17, no. 2, pp. 120-125, 1984.

[7] H. Yu, J. W. Raymonda, T. M. McMahon, and A. A. Campagnari, "Detection of biological threat agents by immunomagnetic microsphere-based solid phase fluorogenic- and electro-chemiluminescence," Biosensors Bioelectronics, vol. 14, no. 10-11, pp. 829-840, 2000.

[8] A. Darko, W. Kabat, N. Constantine, and R. Y. Zhao, "Update on the diagnosis and monitoring of HIV-1 infection," US Infectious Disease, vol. 2007, pp. 2-5, 2007.

[9] M. Sasano, S. Kimura, I. Maeda, and Y. Hidaka, "Analytical performance evaluation of the Elecsys Cyclosporine and Elecsys Tacrolimus assays on the cobas e411 analyzer," Practical Laboratory Medicine, vol. 8, pp. 10-17, 2017.

[10] L. Sommese, C. Sabia, R. Paolillo et al., "Screening tests for hepatitis $B$ virus, hepatitis $C$ virus, and human immunodeficiency virus in blood donors: evaluation of two chemiluminescent immunoassay systems," Scandinavian Journal of Infectious Diseases, vol. 46, no. 9, pp. 660-664, 2014.

[11] O. Jinming, H. Xiaoxu, J. Yangtao, W. Yanan, and S. Hong, “A comparative study on the clinical performance of three fourth generation HIV diagnostic reagents," Chinese Journal of Laboratory Medicine, vol. 36, no. 10, pp. 903-907, 2013.
[12] L. Li, B. Cai, C. Tao, and L. Wang, "Performance evaluation of CLIA forTreponema PallidumSpecific antibodies detection in comparison with ELISA," Journal of Clinical Laboratory Analysis, vol. 30, no. 3, pp. 216-222, 2016.

[13] M. Madiyal, S. Sagar, S. Vishwanath, B. Banerjee, V. K. Eshwara, and K. Chawla, "Comparing assay performance of ELISA and chemiluminescence immunoassay in detecting antibodies to hepatitis B surface antigen," Journal of Clinical and Diagnostic Research: JCDR, vol. 10, no. 11, pp. dc22-dc25, 2016.

[14] A. Sampedro, J. Rodríguez-Granger, C. Gómez, A. Lara, J. Gutierrez, and A. Otero, "Comparative evaluation of a new chemiluminiscent assay and an ELISA for the detection of IgM against measles," Journal of Clinical Laboratory Analysis, vol. 27, no. 6, pp. 477-480, 2013.

[15] T. Wang, D. Li, K. Yan et al., "Performance evaluation of a new fourth-generation HIV Ag/Ab combination electrochemiluminescence immunoassay - evaluation of a new HIV assay," International Journal of STD \& AIDS, vol. 25, no. 4, pp. 267-272, 2013.

[16] S. L. Stramer, R. L. Townsend, G. A. Foster, R. Johnson, B. Weixlmann, and Y. Dodd, "Discordant human T-lymphotropic virus screening with Western blot confirmation: evaluation of the dual-test algorithm for US blood donations," Transfusion, vol. 58, no. 3, pp. 638-640, 2018.

[17] P. Kiely, Y. Stewart, and L. Castro, "Analysis of voluntary blood donors with biologic false reactivity on chemiluminescent immunoassays and implications for donor management," Transfusion, vol. 43, no. 5, pp. 584-590, 2003.

[18] K. Malm, E. Kragsbjerg, and S. Andersson, "Performance of Liaison XL automated immunoassay platform for bloodborne infection screening on hepatitis B, hepatitis C, HIV 1/2, HTLV $1 / 2$ and Treponema pallidum serological markers," Transfusion Medicine, vol. 25, no. 2, pp. 101-105, 2015.

[19] S. Kim, J.-H. Lee, J. Y. Choi, J. M. Kim, and H.-S. Kim, "Falsepositive rate of a "Fourth-Generation" HIV antigen/antibody combination assay in an area of low HIV prevalence," Clinical and Vaccine Immunology, vol. 17, no. 10, pp. 1642-1644, 2010.

[20] P. Kiely and E. Wood, "Can we improve the management of blood donors with nonspecific reactivity in viral screening and confirmatory assays?" Transfusion Medicine Reviews, vol. 19, no. 1, pp. 58-65, 2005.

[21] X. Yang, Y. P. Liu, X. Zhou, C. Gao, and A. Wang, "Investigation report of continuous improvement of clinical blood transfusion records," Chinese Journal of Blood Transfusion, vol. 27, no. 9, pp. 947-949, 2014.

[22] S. B. Lin, Z. X. Zheng, and R. Zhang, "Application and evaluation of chemiluminescence immunoassay in blood screening," Zhongguo Shi Yan Xue Ye Xue Za Zhi, vol. 27, no. 2, pp. 569-572, 2019.

[23] L. Kyunghoon, P. Hyung-Doo, and E.-S. Kang, "Reduction of the HIV seroconversion window period and false positive rate by using ADVIA centaur HIV antigen/antibody combo assay," Annals of Laboratory Medicine, vol. 33, no. 6, pp. 420-425, 2013.

[24] C. Rao, T. Wang, Q. Chen et al., "Performance evaluation of a novel automated HIV Ag/Ab chemiluminescence immunoassay," Clinical Chemistry and Laboratory Medicine (CCLM), vol. 54, no. 9, pp. e255-e258, 2016.

[25] S. Malhotra, N. Marwaha, K. Saluja, and R. R. Sharma, "Improving blood safety using fourth generation HIV ELISA as the screening tool in blood banks - an Indian experience," BMC Infectious Diseases, vol. 12, no. S1, p. 1, 2012.

[26] H. Wang, Y. Yin, and W. Wei, "Evaluation of the 4th ELISA kits for HIV antibody in the STD clinic," China Journal of Leprosy \& Skin Diseases, vol. 26, no. 6, pp. 392-394, 2010. 
[27] P. Nuttall, R. Pratt, L. Nuttall, and C. Daly, "False-positive results with HIV ELISA kits," The Lancet, vol. 328, no. 8505, pp. 512-513, 1986.

[28] P. Liu, Z. Shi, C. Wang et al., "The false-positive and falsenegative predictive value of HIV antibody test in the Chinese population," Journal of Medical Screening, vol. 15, no. 2, pp. 72-75, 2008. 